\title{
Pathophysiology of Osteoarthritis: Evidence against the Viscoelastic Theory
}

\author{
Sharon Dunn ${ }^{a}$ Oleg V. Kolomytkin ${ }^{b}$ Andrew A. Marino ${ }^{b}$ \\ ${ }^{a}$ School of Allied Health Professions, LSU Health Sciences Center, ${ }^{b}$ Department of Orthopaedic Surgery, \\ LSU Health Sciences Center, Shreveport, La., USA
}

\section{Key Words}

Hyaluronan · Synovial fluid · Viscoelasticity · Osteoarthritis

\begin{abstract}
Objectives: Whether changes in the hyaluronan moiety of synovial fluid are associated with osteoarthritis (OA) is unresolved experimentally, notwithstanding frequent statements in the literature that the disease leads to degraded hyaluronan. We evaluated this hypothesis by comparing the molecular weight and concentration of hyaluronan in synovial fluid from patients with and without OA. Methods: Synovial fluid was obtained by needle aspiration from patients with advanced OA (Kellgren-Lawrence Grade IV) and from patients with no radiological or arthroscopic evidence of OA. The distribution of the molecular weight of hyaluronan was measured using both gel electrophoresis and size-exclusion chromatography, and hyaluronan concentration was determined by immunosorbent assay and differential refractometry. Results: The distributions of molecular weight were highly variable within each group of patients; the average distributions, however, were identical in the 2 groups. The average concentration of hyaluronan also did not differ statistically between the 2 groups. Conclusions: We found no evidence of hyaluronan degradation in synovial fluid of pa-
\end{abstract}

tients with OA. Commonly expressed opinion in the literature to the contrary may have resulted from a failure to adequately consider the limitations of previous experimental studies.

Copyright $\odot 2009$ S. Karger AG, Basel

\section{Introduction}

Osteoarthritis (OA) is a degenerative joint disease characterized by the breakdown of articular cartilage. The prevalence of $\mathrm{OA}$ is increasing; for example, in the United States, it will affect 67 million people by 2030 [1]. The development of OA has traditionally been thought to be related to the deterioration of joint synovial fluid [2-7]. In this view, first proposed by Balazs et al. [8], a progressive reduction in the concentration and average molecular weight of the hyaluronan moiety of synovial fluid adversely alters its viscoelastic properties, thereby accounting for or at least contributing to the morbidity of the disease. They recommended exogenous hyaluronan therapy to restore normalcy [9-13], and the practice was approved by many national medical regulatory authorities for treating knee pain in patients with OA [14].

\section{KARGER}

Fax +4161306 1234

E-Mail karger@karger.ch

www.karger.com
(C) 2009 S. Karger AG, Basel

1015-2008/09/0766-0322\$26.00/0

Accessible online at:

www.karger.com/pat
Andrew A. Marino

Department of Orthopaedic Surgery

LSU Health Sciences Center, P.O. Box 33932

Shreveport, LA 71130-3932 (USA)

Tel. +1 318675 6180, Fax +1 318675 6186, E-Mail amarino@lsuhsc.edu 
Hyaluronan, a linear glycosaminoglycan formed by a repeating pair of saccharides, is an important biological signaling molecule [15]; its major receptors are CD44 and RHAMM [16]. We found that CD44 and RHAMM were significantly upregulated in patients with advanced OA [17], and that exogenous hyaluronan downregulated the expression of matrix metalloproteinases, the catabolic enzymes that degrade cartilage [18]. Taken together, these observations suggested to us that dysregulation in hyaluronan signaling was involved in cartilage destruction, and that the mechanism underlying the therapeutic efficacy of exogenous hyaluronan involved normalization of biological signaling rather than restoration of the viscoelastic properties of synovial fluid.

Limitations in both the original study [8] and in many subsequent reports led us to suspect the validity of the view that hyaluronan in patients with OA was abnormal. To facilitate evaluation of the competing theories of the pathophysiology of OA, we directly inquired into whether the putative OA-associated changes in hyaluronan would be observed when some of the limitations were taken into consideration. Our objectives were to compare the concentration and average molecular weight of hyaluronan in synovial fluids obtained from the knees of patients with advanced OA with the results obtained from patients who had no radiological or arthroscopic evidence of the disease.

\section{Methods}

\section{Study Design}

The number of disaccharides in a hyaluronan molecule in synovial fluid varies from 1 to more than 25,000. The intersubject variability in number of molecules of each molecular size (the factor that determines how many subjects are needed to demonstrate a statistical difference between study groups) was unknown. We initially designed this study with 5 subjects in each study group, intending to add additional subjects, if justified.

To maximize the possibility of finding degraded hyaluronan in association with OA, we studied patients who had severe disease necessitating total joint arthroplasty. To exclude the presence of OA in the control group, we used X-rays and arthroscopy (the best available methods) to rule out the presence of the disease. To insure the reliability of the measurements of molecular weight and concentration, each parameter was measured using 2 independent methods.

\section{Synovial Fluid}

The study patients came from a single-site, knee-only orthopedic practice (D.D.W.). Synovial fluid $(0.5-1 \mathrm{ml})$ was obtained by needle aspiration from 5 patients immediately before they underwent total knee arthroplasties for advanced OA (grade IV, Kellgren-Lawrence scale); the average age was 71 years (range 53-
85). Control synovial fluid was obtained from 5 patients (average 29 years, range 19-47) undergoing meniscectomy or ligament reconstruction who had no radiological evidence of OA and no arthroscopic evidence of OA or inflammation of the synovium (control patients). The fluid samples were centrifuged at $4,500 \mathrm{~g}$ for $10 \mathrm{~min}$ at $4{ }^{\circ} \mathrm{C}$ and stored at $-80^{\circ} \mathrm{C}$ until analyzed. In preliminary experiments we established that freezing had no effect on the parameters measured. All experimental procedures were approved by the review board for human research at the LSU Health Sciences Center.

\section{Molecular Weight}

The weight distribution of hyaluronan molecules was measured by gel electrophoresis and by size-exclusion chromatography coupled to multiangle laser light scattering (SEC-MALLS). For electrophoresis, all samples of synovial fluid were initially treated with pronase to remove proteins [19]. Treated samples containing $7 \mu \mathrm{g}$ of hyaluronan $(0.5 \mathrm{mg} / \mathrm{ml}$ in TAE buffer with $2 \mathrm{M}$ sucrose) were electrophoresed ( $0.5 \%$ agarose) at room temperature. Five gels were run, each of which contained duplicate samples from $1 \mathrm{OA}$ and 1 non-OA (control) patient, and hyaluronan molecular-weight markers; the hyaluronan lanes were separated by empty lanes to permit measurement of the gel background. The hyaluronan was detected by staining the gel overnight $(0.005 \%$ Stains-All in $50 \%$ ethanol). The stained material was identified as hyaluronan by running additional gels that had been treated with Streptomyces hyaluronate lyase; we found that all the material ran off the gel, indicating that the molecular weights of the digested fragments were $<27 \mathrm{kDa}$.

The gels were photographed using a digital camera, after verifying in control experiments that the optical density (OD) response of the camera was linear over the range of dye concentrations used. The OD of each lane was measured (ImageQuant; Molecular Dynamics, Amersham, Piscataway, N.J., USA) as a function of the migration distance on the gel. For each OA and each control lane, the distributions (OD versus migration distance) of the adjacent pair of empty lanes were averaged and then subtracted from the distribution measured in the synovial-fluid lane. The duplicate measurements of the synovial-fluid samples were averaged and the ordinate for the hyaluronan distribution curve was calibrated by measuring the area under the curve of OD versus the migration distance and setting the area equal to $7 \mu \mathrm{g}$ of hyaluronan (the amount of hyaluronan loaded on the gel). The migration positions of the molecular-weight hyaluronan standards were plotted versus inverse log of hyaluronan weight and fitted by linear regression analysis to produce the calibration curve (separate curves for each gel), which was used to establish and label the abscissa for the hyaluronan distribution curve. The weight-average molecular weight of hyaluronan was calculated directly from the distribution curve. The overall results of the study were the same when the number-average molecular weight was used.

For determination of molecular-weight distribution by SECMALLS, the synovial-fluid samples were diluted by a factor of 20 (v/v) using elution buffer (50 mM NaPO$, 150 \mathrm{mM} \mathrm{NaCl}, \mathrm{pH} 7)$, and incubated with pronase $(30 \mathrm{U} / \mathrm{ml})$ at $37^{\circ} \mathrm{C}$ for $2 \mathrm{~h}$. After the enzymes were heat inactivated $\left(100^{\circ} \mathrm{C}\right.$ for $\left.4 \mathrm{~min}\right)$, the samples were centrifuged, and the supernatant was transferred to an autoinjector. Chromatographic separation of samples was performed at a flow rate of $0.475 \mathrm{ml} / \mathrm{min}$ at $22^{\circ} \mathrm{C}$ using 2 or 3 Plaquagel-OH 
columns in series (Polymer Laboratories, Amherst, Mass., USA). Multiangle laser light scattering analysis and interferometric refractometry were performed continuously on the column eluate (Dawn DSP, OPTILAB DSP; Wyatt Technologies Inc., Santa Barbara, Calif., USA). The molecular mass of hyaluronan was calculated from the SEC-MALLS data using a commercial software program (Astra; Wyatt Technology Corporation, Santa Barbara, Calif., USA). Further details are given elsewhere [20].

\section{Concentration}

Hyaluronan concentration in synovial fluid was determined by immunoabsorbent assay (Corgenix, Denver, Colo., USA), and by differential refractometry (Wyatt Technology).

\section{Reagents}

Select hyaluronan standards $(27-6,100 \mathrm{kDa})$ were obtained from Hyalose (Oklahoma City, Okla., USA). Pronase (Streptomyces griseus, nuclease-free, specific activity, $183 \mathrm{PUK} / \mathrm{mg}$ ) was obtained from Calbiochem (San Diego, Calif., USA). All other reagents were obtained from Sigma-Aldrich (St. Louis, Mo., USA).

\section{Statistics}

The measurements from the 2 groups were compared using the t test. The relation between hyaluronan concentration and the age of the patient was evaluated using the Pearson correlation coefficient.

\section{Results}

Electrophoresis revealed the presence of a continuous distribution of hyaluronan polymer size in each patient, as expected (fig. 1). The distributions of molecular weight were highly variable within both the OA and control groups (fig. 2a, b, respectively); the averages of the distributions, however, were nearly identical (fig. 2c). The computed average molecular weights were $7.7 \pm 1.5$ and $6.3 \pm 2.1 \mathrm{MDa}$ for the $\mathrm{OA}$ and control groups, respectively.

Small hyaluronan molecules are pro-inflammatory but large molecules are anti-inflammatory [21], so we compared average distributions in the tail region (fig. 2; $<1 \mathrm{MDa}$ ). The average concentrations in the tail ( $\pm \mathrm{SD}$ ) were $0.83 \pm 0.41 \mathrm{ng} / \mathrm{ml}$ and $0.77 \pm 0.39 \mathrm{ng} / \mathrm{ml}$ for the $\mathrm{OA}$ and control groups, respectively $(\mathrm{p}>0.05)$.

When the distributions of hyaluronan molecular weight in synovial fluid were determined by SEC-MALLS, large variations were again seen with each group (fig. 3a, b), and again the average curves were identical within 1 SD (fig. 3c).

Hyaluronan concentration was $1.4 \pm 0.1$ and $1.8 \pm$ $0.3 \mathrm{mg} / \mathrm{ml}$ in the OA and control groups, respectively, as determined using immunoabsorbent assay $(p>0.05)$. The corresponding values found by means of differential

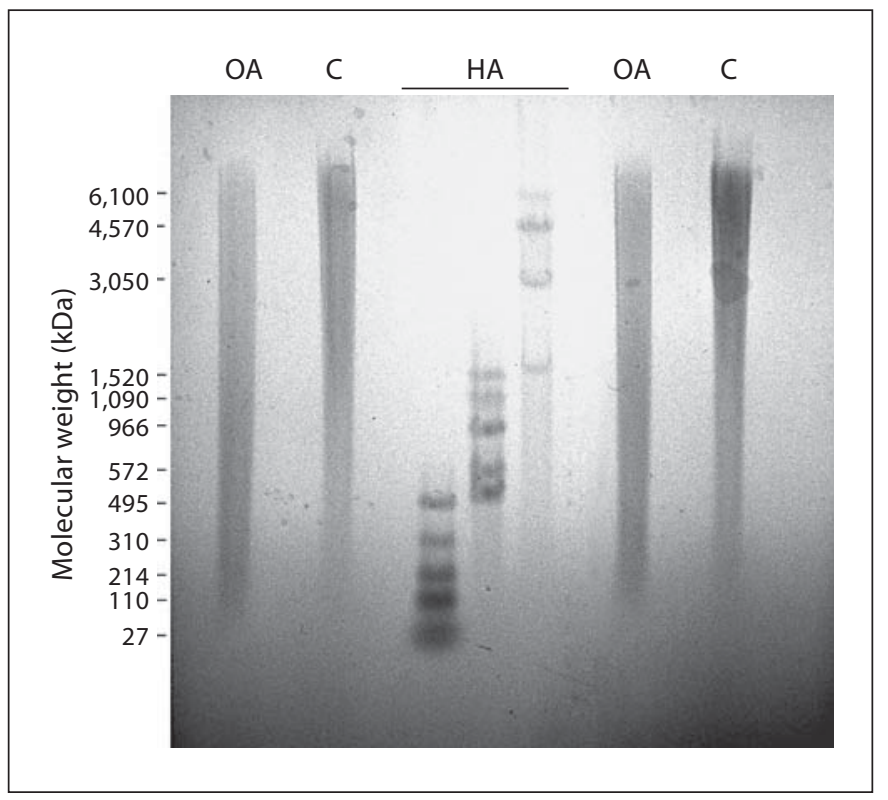

Fig. 1. Typical agarose gel of synovial fluid samples from 1 patient with OA and 1 patient without the disease (C), each run in duplicate; the patient pairing was arbitrary. Adjacent empty lanes were used for determinations of local gel background. HA = Hyaluronan molecular-weight standards.

refractometry were $1.3 \pm 0.1$ and $1.9 \pm 0.4 \mathrm{mg} / \mathrm{ml}(\mathrm{p}>$ $0.05)$. Patient age was highly correlated with hyaluronan concentration within each experimental group, as determined by both methods of measurement (fig. 4).

\section{Discussion}

Correct assessment of the pathophysiology of OA is critical for the development of disease-modifying drugs. Balazs et al. [8] studied synovial fluids from hundreds of normal and osteoarthritic subjects and concluded that the morbidity of the disease arose from compromised viscoelastic properties of the synovial fluid caused by hyaluronan degradation. That viewpoint was repeatedly emphasized and offered as the rationale for injecting synthetic hyaluronan into the joints of patients suffering from OA-related pain [9-13]. The clinical success of the treatment in relieving pain [14] was taken as confirmatory evidence of the proposed pathoetiology [10-12], but for several reasons we suspected that the theory was incorrect and that the clinical results were only fortuitous. First, in their seminal study, the investigators pooled the synovial fluids, and did not obtain clinical evidence of 


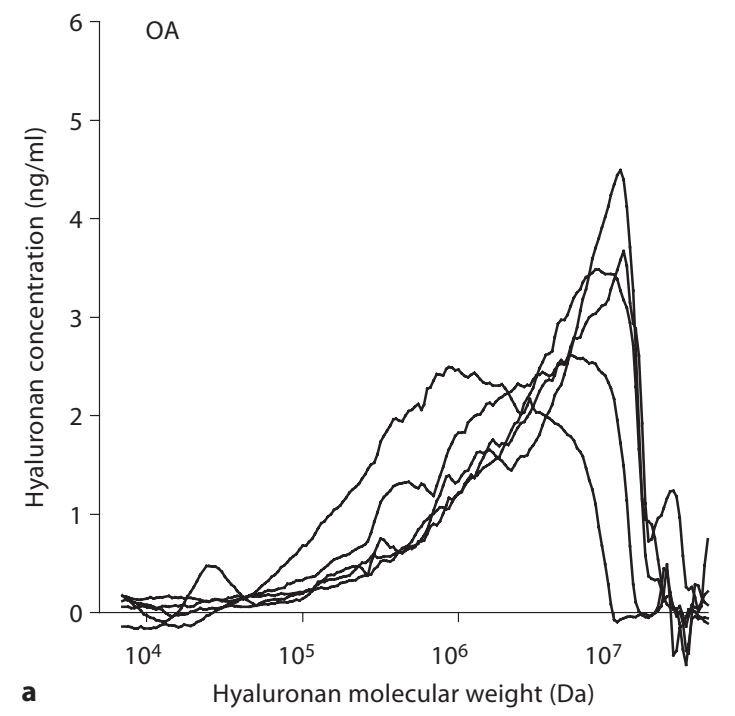

Fig. 2. Distribution of the molecular weight of the hyaluronan in the synovial fluid of patients with (a) and without (b) OA, determined by gel electrophoresis. $\mathrm{n}=5$ in each group. $\mathbf{c}$ Average distribution in each group.

the presence or severity of the OA; the experimental conditions therefore could not permit drawing a reliable inference of association between synovial-fluid changes and the presence of OA. Second, on balance, subsequent studies by other investigators failed to support the putative association between OA and hyaluronan degradation. For example, although the average molecular weight of hyaluronan in synovial fluid from normal joints varied from 2.75 to $10.9 \mathrm{MDa}$, compared with 1.8 to $2.1 \mathrm{MDa}$ in synovial fluid from patients with OA [8, 22-25], each study reported results from patients with or without OA, but the 2 groups were not directly compared in the same study. Similarly, although the average concentration of hyaluronan in synovial fluid (weighted by number of patients) was $1.7 \mathrm{mg} / \mathrm{ml}$ in patients with $\mathrm{OA}[8,23,26-32]$,
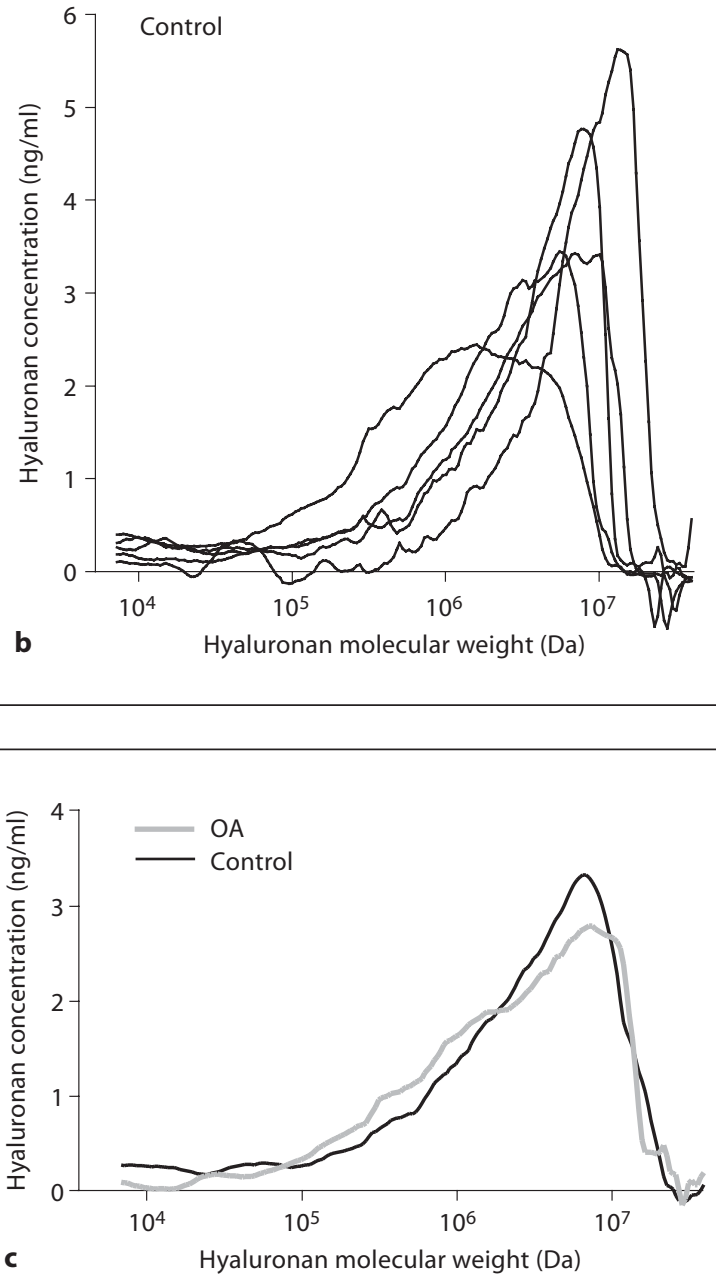

and $2.5 \mathrm{mg} / \mathrm{ml}$ in the controls, the variance in the data was too great to permit accepting the difference as statistically valid. Still worse, almost none of the studies considered the possible confounding effects of the age of the subject. On the other hand, ample research established that hyaluronan concentration decreased with age. For example, the average concentration in 36 normal subjects aged $70-79$ was $74 \%$ of the corresponding value obtained from 29 subjects aged 20-29 [33]. The average concentration in 10 subjects aged $70-79$ was $74 \%$ of the corresponding value found in 22 subjects aged 20-29 [30]. Moreover, the many different methods used to measure hyaluronan concentration had varying degrees of sensitivity, and none of the studies employed a second, independent method to verify the original result. Finally, the visco- 


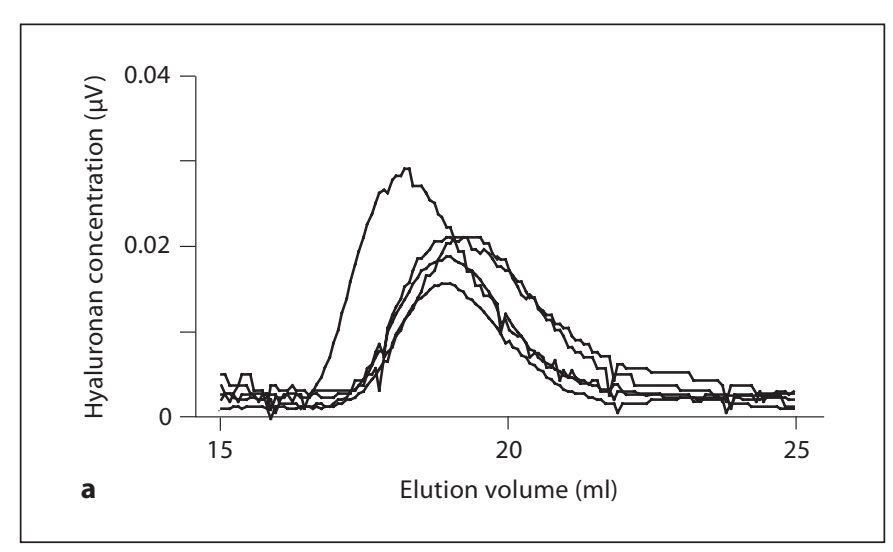

Fig. 3. Distribution of the molecular weight of the hyaluronan in the synovial fluid of patients with (a) and without (b) OA, determined by size-exclusion chromatography and laser light scattering. $\mathrm{n}=5$ in each group. c Average distribution in each group. Insert shows variation in the $\mathrm{SD}$ of the average distributions.

elastic theory, which was first proposed prior to the development of cellular and molecular biology [8], is not a theory in the modern sense because it does not describe a mechanism by which viscoelastic changes could be transduced into a biological signal.

Our aim was to characterize and compare the molecular weight and concentration of hyaluronan in synovial fluids from patients who did or did not have OA, while taking into consideration some of the shortcomings we perceived in the earlier studies. In particular, we standardized the severity of the disease in the patients from whom synovial-fluid samples were obtained, ensured that OA was not present in the controls, used optimal measurement methods to characterize the study variables, employed 2 independent measurement methods for each parameter, and analyzed the results statistically. There were, however, 2 related but distinct limitations in the previous studies that, for ethical reasons and because of a natural relation between OA and ageing, we could not overcome in the present study (see below).
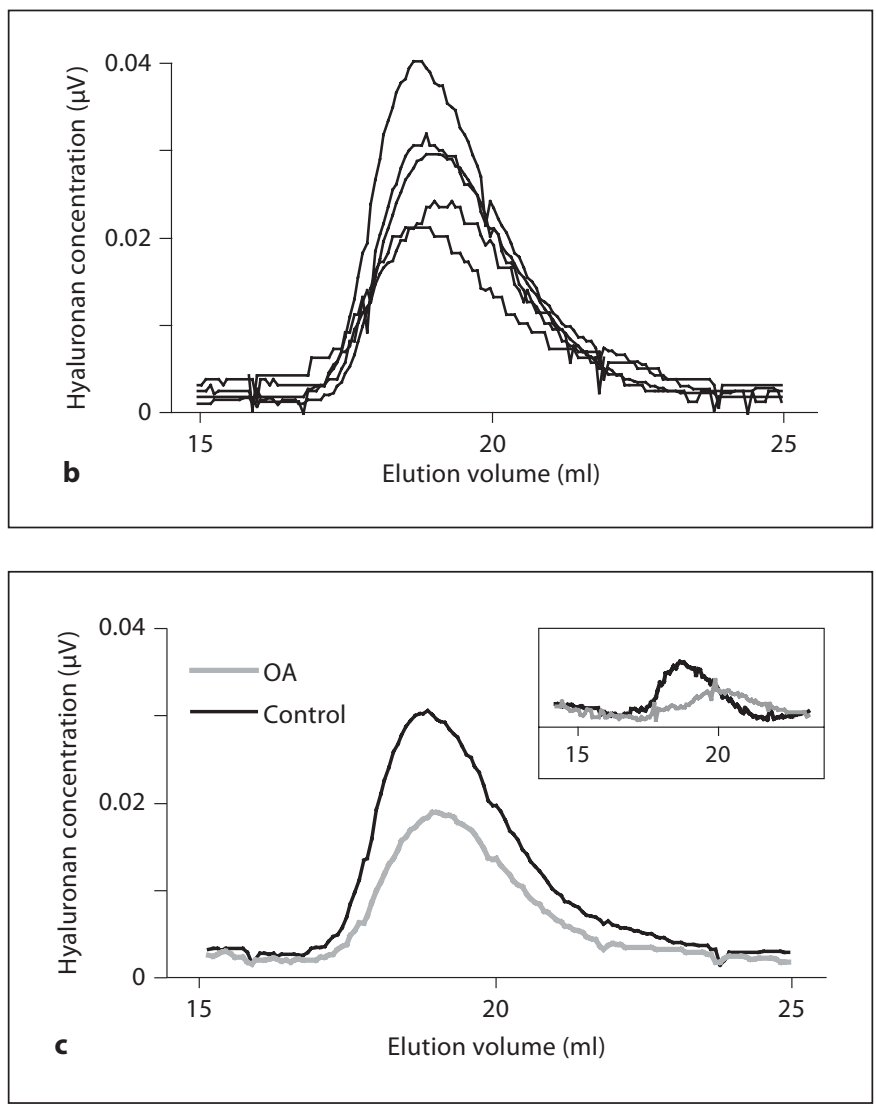

The average distributions of hyaluronan varied widely within each group of patients, as determined by each of 2 independent measurement methods (fig. 2,3). The extent of the variability within each group, which had not previously been reported, clearly indicated that hyaluronan molecular weight was a poor parameter for evaluating the causes of OA, because any specific value was as likely as not to be associated with the presence of the disease. Including additional subjects in the study was unnecessary because the likely result would have been to reinforce the virtual identity of the average distributions (fig. 2c, 3c).

Using immunoabsorbent assays, the concentrations of hyaluronan in the patients with OA was $1.4 \pm 0.1 \mathrm{mg} / \mathrm{ml}$, compared with $1.8 \pm 0.3 \mathrm{mg} / \mathrm{ml}$ in the controls; essentially identical results $(1.3 \pm 0.1$ and $1.9 \pm 0.4)$ were found using refractometry. The patients with OA had an average age of 71 years, compared with an average age of 29 years in the control subjects. Hyaluronan concentration in normal subjects is known to be inversely related to patient age [30,33]. Consequently, even assuming that the difference between groups became statistically sig- 

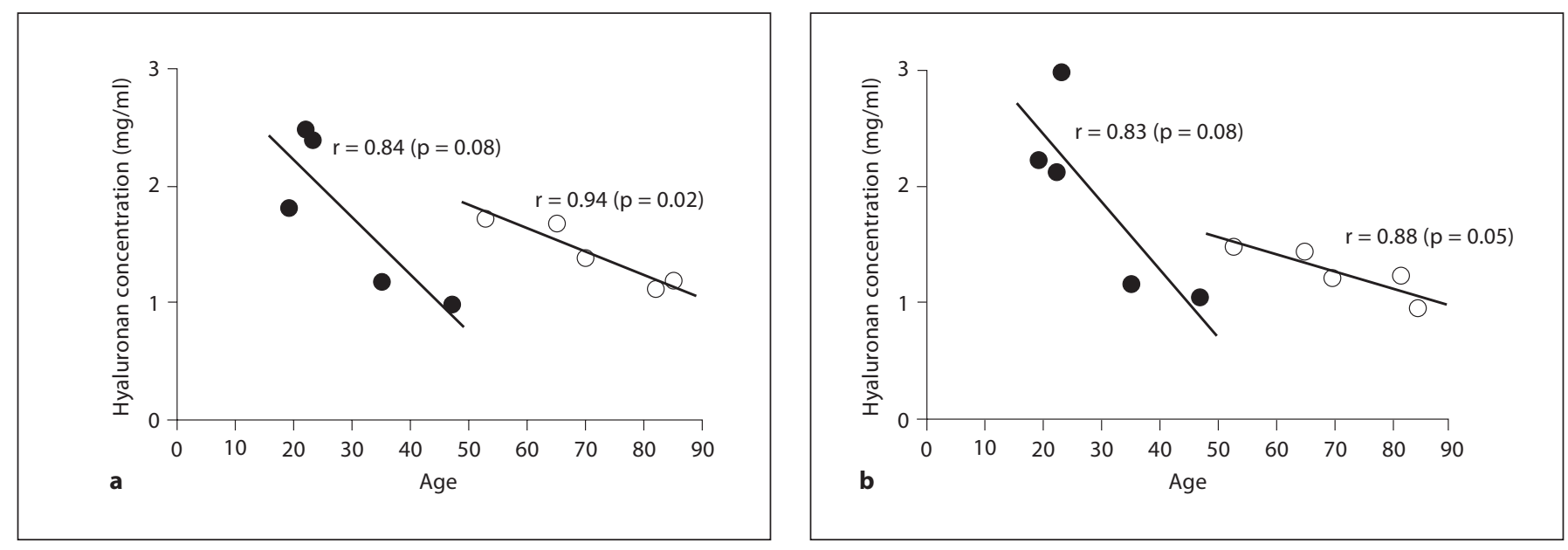

Fig. 4. Relationship between patient age and hyaluronan concentration in synovial fluid as determined by immunoabsorbent assay (a) and differential refractometry (b). Solid (open) circles, OA (control) patients. r, Pearson correlation coefficient.

nificant if hyaluronan concentration were measured in additional patients, the concentration differences could be explained more parsimoniously on the basis of a confounding effect of age, rather than the presence of the disease. This idea was supported by our finding that patient age was inversely correlated with hyaluronan concentration in both patient groups (fig. 4). Overall, our results were inconsistent with expressions of common wisdom to the effect that a link existed between degradative changes in hyaluronan and the presence of OA [2-7]; we found no evidence of a link.

Our conclusion should be understood in the light of 2 particular limitations. Ideally, the control group should have been age-matched and clinically normal (rather than simply free of OA, as was the case here). However, it is not possible to employ such controls because patients age-matched to those with grade IV disease invariably have some degree of OA; further, even if normal agematched subjects could be found, there are serious ethical problems associated with obtaining synovial fluid from clinically normal subjects simply for experimental purposes. For these reasons, the existence of a relationship between $\mathrm{OA}$ and the concentration of hyaluronan in synovial fluid probably cannot be addressed experimentally. In retrospect, we can see that the original hypothesis [8] was stillborn because it could never actually be tested using appropriate controls.

Synovial fluid from patients with OA contains aggregated and monomeric proteoglycans, as well as other constituents including proteins and cells [34]. We did not address the issue of what role these substances might play in relationship to OA.

Hyaluronan is a signaling molecule [21] and might therefore influence the course of the disease via changes in hyaluronan receptors. This theory is consistent with our observations that CD44 and RHAMM, the 2 principal hyaluronan receptors, were overexpressed in patients with OA [17].

We found no evidence of hyaluronan degradation in synovial fluid of patients with OA. Commonly expressed opinion in the literature to the contrary, it probably resulted from a failure to adequately consider the limitations of previous experimental studies.

\section{Conclusions}

We found no evidence of hyaluronan degradation in synovial fluid of patients with OA. Commonly expressed opinion in the literature to the contrary may have resulted from a failure to adequately consider the limitations of previous experimental studies. 


\section{References}

1 Hootman JM, Helmick CG: Projections of US prevalence of arthritis and associated activity limitations. Arthritis Rheum 2006;54: 226-229.

2 Insall JN, Scott WN: Surgery of the Knee, ed 3. New York, Churchill Livingstone, 2001.

3 Jones A, Doherty M: Intra-articular therapies in osteoarthritis; in Brandt KD, Doherty M, Lohmander LS (eds): Osteoarthritis. Oxford, Oxford Press, 1998, pp 299-306.

4 Jüni P, Reichenbach S, Trelle S, Tschannen B, Wandel S, Jordi B, Züllig M, Guetg R, Häuselmann HJ, Schwarz H, Theiler R, Ziswiler HR, Dieppe PA, Villiger PM, Egger M, Swiss Viscosupplementation Trial Group: Efficacy and safety of intraarticular hylan or hyaluronic acids for osteoarthritis of the knee. A randomized controlled trial. Arthritis Rheum 2007;56:3610-3619.

5 Mathieu P, Conrozier T, Vignon E, Rozand Y, Rinaudo M: Rheologic behavior of osteoarthritic synovial fluid after addition of hyaluronic acid. A pilot study. Clin Orthop Relat Res DOI: 10.1007/s11999-11009-10867-x.

6 Strauss EJ, Hart JA, Miller MD, Altman RD, Rosen JE: Hyaluronic acid viscosupplementation and osteoarthritis. Current uses and future directions. Am J Sports Med DOI: 10.1177/0363546508326984.

-7 Watterson JR, Esdaile JM: Viscosupplementation: Therapeutic mechanisms and clinical potential in osteoarthritis of the knee. J Am Acad Orthop Surg 2000;8:277-284.

8 Balazs EA, Watson D, Duff IF, Roseman S: Hyaluronic acid in synovial fluid. I: Molecular parameters of hyaluronic acid in normal and arthritis human fluids. Arthritis Rheum 1967;10:357-376.

9 Balazs EA: The physical properties of synovial fluid and the special role of hyaluronic acid; in Helfet AJ (ed): Disorders of the Knee. Philadelphia, J.B. Lippincott Co., 1982, pp 61-74.

$\checkmark 10$ Balazs EA: Viscosupplementation for treatment of osteoarthritis: From initial discovery to current status and results. Surg Technol Int 2004;12:278-289.

11 Balazs EA, Denlinger JL: The role of hyaluronic acid in arthritis and its therapeutic use; in Peyron JG (ed): Osteoarthritis - Current Clinical and Fundamental Problems. Basel, Geigy, 1985, pp 165-174.
12 Balazs EA, Denlinger JL: Viscosupplementation: a new concept in the treatment of osteoarthritis. J Rheumatol 1993;20:309.

13 Peyron JG, Balazs EA: Preliminary clinical assessment of $\mathrm{Na}$-hyaluronate injection into human arthritic joints. Pathol Biol (Paris) 1974;22:731-736.

14 Bellamy N, Campbell J, Robinson V, Gee T, Bourne R, Wells G: Viscosupplementation for the treatment of osteoarthritis of the knee. Cochrane Database Syst Rev 2006: CD005321.

15 Itano N: Simple primary structure, complex turnover regulation and multiple roles of hyaluronan. J Biochem 2008; 144:131-137.

16 Slevin M, Krupinski J, Gaffney J, Matou S, West D, Delisser H, Savani RC, Kumar S: Hyaluronan-mediated angiogenesis in vascular disease: uncovering RHAMM and CD44 receptor signalling pathways. Matrix Biol 2007;26:58-68.

17 Dunn S, Kolomytkin OV, Waddell DD, Marino AA: Hyaluronan-binding receptors: possible involvement in osteoarthritis. Mod Rheumatol DOI: 10.1007/s10165-008-0136-y.

18 Waddell DD, Kolomytkin OV, Dunn S, Marino AA: Hyaluronan suppresses IL-1 $\beta$-induced metalloproteinase activity from synovial tissue. Clin Orthop 2007;465:241-248.

19 Lee HG, Cowman MK: An agarose gel electrophoretic method for analysis of hyaluronan molecular weight distribution. Anal Biochem 1994;219:278-287.

20 Baggenstoss BA, Weigel PH: Size exclusion chromatography - multiangle laser light scattering analysis of hyaluronan size distributions made by membrane-bound hyaluronan synthase. Anal Biochem 2006;352:243251.

21 Cantor JO, Nadkarni PP: Hyaluronan: The Jekyll and Hyde molecule. Inflamm Allergy Drug Targets 2006;5:257-260.

22 Altman RD: Intra-articular sodium hyaluronate in osteoarthritis of the knee. Semin Arthritis Rheum 2000;30:11-18.

23 Castor CW, Prince RK, Hazelton MJ: Hyaluronic acid in human synovial effusions; a sensitive indicator of altered connective tissue cell function during inflammation. Arthritis Rheum 1966;9:783-794.
24 Dahl LB, Dahl IM, Engström-Laurent A, Granath K: Concentration and molecular weight of sodium hyaluronate in synovial fluid from patients with rheumatoid arthritis and other arthropathies. Ann Rheum Dis 1985;44:817-822.

25 Evanich JD, Evanich CJ, Wright MB, Rydlewicz JA: Efficacy of intraarticular hyaluronic acid injections in knee osteoarthritis. Clin Orthop 2001;390:173-181.

26 Grimshaw J, Trocha-Grimshaw J, Fisher W, Rice A, Smith S, Spedding P, Duffy J, Mollan $\mathrm{R}$ : Quantitative analysis of hyaluronan in human synovial fluid using capillar electrophoresis. Electrophoresis 1996;17:396-400.

27 Kofoed J, Tocci AA, Barcelo AC: The acidic glycosaminoglycans of the synovial fluid in rheumatoid arthritis. Experientia 1973;29: 680-681.

28 Mazzucco MD, Scott R, Spector M: Composition of joint fluid in patients undergoing total knee replacement and revision arthroplasty: correlation with flow properties. Biomaterials 2004;25:4433-4445.

-29 Miyaguchi M, Kobayashi A, Kadoya Y, Ohashi H, Yamano Y, Takaoka K: Biochemical change in joint fluid after isometric quadriceps exercise for patients with osteoarthritis of the knee. Osteoarthritis Cartilage 2003;11:252-259.

30 Nakayama Y, Narita T, Mori A, Uesaka S, Miyazaki K, Ito $\mathrm{H}$ : The effects of age and sex on chondroitin sulfates in normal synovial fluid. Arthritis Rheum 2002;46:2105-2108.

31 Pitsillides AA, Will RK, Bayliss MT, Edwards JCW: Circulating and synovial fluid hyaluronan levels. Arthritis Rheum 1994;7: 1030-1038.

32 Saito T, Takeuchi R, Mitsuhashi S, Uesugi M, Yoshida T, Koshino T: Use of joint fluid analysis for determining cartilage damage in osteonecrosis of the knee. Arthritis Rheum 2002;46:1813-1819.

33 Uesaka S, Miyazaki K, Ito H: Age-related changes and sex differences in chondroitin sulfate isomers and hyaluronic acid in normal synovial fluid. Mod Rheumatol 2004;14: 470-475.

34 Carroll GJ: Spectrophotometric measurement of proteoglycans in osteoarthritic synovial fluid. Ann Rheum Dis 1987;46:375379 . 